\title{
Síndrome hepatopulmonar e hipertensión portopulmonar: Dos entidades a diferenciar
}

\author{
MÓNICA ZAGOLÍN B.*, JUAN NICOLÁS MEDEL F.** y JOSÉ VALERA M.***
}

\section{Hepatopulmonary syndrome and portopulmonary hypertension: Two distinct entities}

The clinical course of patients with portal hypertension or liver disease may be complicated by two low prevalence entities with high morbimortality: the hepatopulmonary syndrome (HPS) and portopulmonary hypertension (PPHT). Each one is a consequence of an impaired hepatic clearance of several vascular mediators, triggering vasodilation of the pulmonary vascular territory in HPS and vasoconstriction with vessel remodelation in PPHT. Both disorders have some similar clinical findings, but useful findings for differential diagnosis are the presence of platypnoea and orthodeoxia in HPS, and echocardiographic extracardiac and intrapulmonary shunt in HPS or pulmonary hypertension in PPHT. Currently, liver transplantation is the only effective treatment for both entities provided that indication and timing must be accurately evaluated. We present a review and three cases of both entities.

Key words: Hepatopulmonary syndrome, portopulmonary hypertension, liver cirrhosis, portal hypertension.

\section{Resumen}

El curso clínico de los pacientes con cirrosis y/o hipertensión portal puede verse complicado por dos entidades de baja prevalencia pero de elevada morbimortalidad, que corresponden al síndrome hepatopulmonar (SHP) y la hipertensión portopulmonar (HPP). Ambas se presentan a consecuencia de un déficit en la depuración hepática de diversos mediadores vasculares, provocando en el territorio pulmonar una vasodilatación en el SHP y una vasoconstricción con remodelación vascular en la HPP. Si bien estas entidades comparten algunos aspectos clínicos, resulta útil en su diferenciación la presencia de platipnea y ortodeoxia y el hallazgo ecocardiográfico de un shunt extracardíaco e intrapulmonar en el SHP, o de hipertensión pulmonar en HPP. Hasta el momento la única terapia efectiva para ambas entidades es el trasplante hepático, cuya indicación exige una evaluación rigurosa y oportuna. Se presenta una revisión y tres casos clínicos de ambas entidades.

Palabras clave: Síndrome hepatopulmonar, hipertensión portopulmonar, cirrosis hepática, hipertensión portal.

Abreviaturas

DHC : Daño hepático crónico.

DLCO : Estudio de difusión con monóxido de carbono.

eNOS : Sintetasa endotelial de óxido nítrico.

EPOC : Enfermedad pulmonar obstructiva crónica.
ET-1 : Endotelina-1.

HPA : Hipertensión pulmonar arterial.

HPP : Hipertensión portopulmonar.

iNOS : Sintetasa inducible de óxido nítrico.

NO : Óxido nítrico.

$\mathrm{PA}-\mathrm{aO}_{2}$ : Diferencia alvéolo-arterial de oxígeno

PAOP : Presión de capilar pulmonar o presión de oclusión.

* Médico Broncopulmonar, Departamento de Medicina, Instituto Nacional del Tórax. Profesor Asistente, Facultad de Medicina, Universidad de Chile.

** Interno de Medicina. Departamento de Medicina Oriente, Universidad de Chile, Hospital del Salvador.

*** Médico Gastroenterólogo, Hepatólogo, miembro del equipo de trasplante hepático del Hospital Clínico de la Universidad de Chile. 
PAPM : Presión arterial pulmonar media.

PAPS : Presión arterial pulmonar sistólica.

RVP : Resistencia vascular pulmonar.

SHP : Síndrome hepatopulmonar.

TH : Trasplante hepático ortotópico.

TIPS : Cortocircuito porto-sistémico por vía transyugular.

TNF- $\alpha$ : Factor de necrosis tumoral alfa.

\section{Introducción}

El curso clínico de los pacientes con cirrosis hepática y/o hipertensión portal puede verse complicado por dos entidades de baja prevalencia, pero elevada morbimortalidad, que corresponden al síndrome hepatopulmonar (SHP) y la hipertensión portopulmonar (HPP) que han cobrado especial interés en la actualidad en relación al éxito del trasplante hepático ortotópico (TH). El primero se debe a una dilatación vascular intrapulmonar que genera un defecto en la oxigenación arterial y el segundo al aumento de la resistencia vascular pulmonar (RVP) como consecuencia de la obstrucción del flujo a nivel del lecho arteriolar pulmonar ${ }^{1}$.

Aunque sean infrecuentes, es importante que el clínico reconozca estas dos entidades asociadas al paciente con daño hepático crónico, debido al curso progresivo y de mal pronóstico que desarrollan sin tratamiento, y las diferencie de otras condiciones que suelen acompañar al paciente con enfermedad hepática avanzada tales como atelectasia, neumonía, congestión pulmonar o hidrotórax hepático ${ }^{1,2}$. Si bien ambas tienen en común la presencia de hipertensión portal y shunt portosistémico y el trasplante hepático es su tratamiento definitivo, existe un profundo contraste en la patogenia involucrada que lleva a una expresión clínica y una aproximación diferente en cada una de ellas.

Para facilitar la labor del clínico en diferenciar y tratar oportunamente ambas entidades es que se describen en detalle cada una de ellas a la luz de la información actual y se presentan casos clínicos ilustrativos.

\section{Síndrome hepatopulmonar}

El síndrome hepatopulmonar (SHP), descrito en 1884 por Fluckiger $^{3}$, es una causa de hipoxemia en pacientes con enfermedad hepática, debido a una vasodilatación intrapulmonar anormal, que conduce a un exceso de perfusión en relación a la ventilación ${ }^{4}$.
Generalmente, esta entidad se encuentra en pacientes con cirrosis hepática clínica, funcional e histológica, afectando entre un $4 \mathrm{y}$ un $30 \%$ de estos pacientes ${ }^{1,4}$. No se ha observado una correlación entre el grado de hipoxemia y el nivel de daño hepático ${ }^{5}$, pero el riesgo parece ser mayor en los pacientes clasificados como Child $\mathrm{C}^{6}$.

\section{Definición}

El SHP ha sido clásicamente definido por la tríada de enfermedad hepática avanzada, vasodilatación intrapulmonar localizada o difusa, e hipoxemia con aumento de la diferencia alvéoloarterial de oxígeno $\left(\mathrm{P}_{\mathrm{A}-\mathrm{a}} \mathrm{O}_{2}\right)$ respirando aire ambiental (> $15 \mathrm{~mm} \mathrm{Hg}$ en los menores de 64 años y > $20 \mathrm{mmHg}$ en los mayores de 64 años) $)^{1,5}$. Sin embargo, esta definición es incompleta, ya que el síndrome se ha visto asociado a hipertensión portal sin cirrosis como es en los casos de trombosis de la vena porta, fibrosis hepática congénita o síndrome de Budd-Chiari ${ }^{1}$. En relación a lo anterior parece más operativo definir el SHP por la coexistencia de disnea, cianosis, platipnea (disnea que aumenta en posición erecta), ortodeoxia (desaturación $>5 \%$ o caída $>4$ mmHg de $\mathrm{PO}_{2}$ al pasar desde posición supina a erecta) y posible hipocratismo digital; todos a consecuencia de la hipertensión portal y el shunt portosistémico ${ }^{1}$.

\section{Etiopatogenia}

La etiología del SHP no está aclarada pero se ha encontrado una mayor correlación con la hipertensión portal que con el daño hepático en si $^{7}$. La marca patogénica del SHP es la dilatación microvascular arterial pulmonar, que puede resultar de un tono arteriolar pre-capilar disminuido e involucrando, además, mecanismos de angiogénesis y remodelación ${ }^{5}$ (Figura 1). Se ha planteado que la vasodilatación a nivel pulmonar se debe al incremento de la producción vascular de moléculas vasodilatadoras, particularmente del óxido nítrico $(\mathrm{NO})^{8}$. Se ha descrito casos en que el aumento del NO exhalado se normaliza luego del trasplante hepático ${ }^{9}$. Además, se han comunicado casos aislados de mejoría transitoria con inhibidores de la producción de $\mathrm{NO}(\mathrm{N}-$ nitro-L-Arginina metil ester) ${ }^{10} \mathrm{o}$ de su acción (azul de metileno $)^{11}$. No se conoce con exactitud el mecanismo del incremento del NO en estos pacientes pero, basado en un modelo animal de cirrosis hepática y SHP, se cree que existe un aumento de la producción de endotelina-1 (ET-1) en el hígado y en el epitelio biliar, que aumentaría la expresión de la enzima NO sintetasa 
endotelial (eNOS) en la circulación pulmonar, mediante la activación de receptores de ET-1 tipo B. En este SHP experimental, se ha observado además una acumulación de macrófagos intravasculares, los cuales transitoriamente producen NO sintetasa inducible (iNOS) y, progresivamente, hemoxigenasa tipo 1 , agregándose además monóxido de carbono como agente patogénico $^{12}$. Se ha observado que la disminución del factor de necrosis tumoral-alfa (TNF- $\alpha$ ), ya sea bloqueando su producción con pentoxifilina o disminuyendo la traslocación bacteriana y de exotoxinas, presente en la cirrosis, se previene la acumulación de macrófagos y el aumento de la iNOS con lo que se atenúa el SHP ${ }^{7}$. En definitiva, el trastorno fisiopatológico del SHP se debería a un tono vascular anormal con pérdida de la auto-regulación del flujo pulmonar y vasodilatación extrema.

\section{Mecanismos de hipoxemia}

No existe un mecanismo único que explique la hipoxemia en este síndrome. Los tres determinantes intrapulmonares de la hipoxemia son: la disminución de la relación ventilación-perfusión (V/Q), la presencia de shunt intrapulmonar y la alteración en la difusión de oxígeno, que explican la platipnea y ortodeoxia (Figura 2). La alteración $\mathrm{V} / \mathrm{Q}$, por la existencia de regiones pulmonares normalmente ventiladas pero sobreperfundidas, es clave para comprender el trastorno del intercambio gaseoso. Por otro lado, la difusión se encuentra también alterada, presumiblemente porque al encontrarse los capilares pulmonares dilatados, aumenta la distancia entre alvéolo y glóbulo rojo dificultando el equilibrio de gases, especialmente en estados hiperdinámicos propios del DHC. En estadios iniciales, el desequilibrio V/Q puede ser leve y el shunt

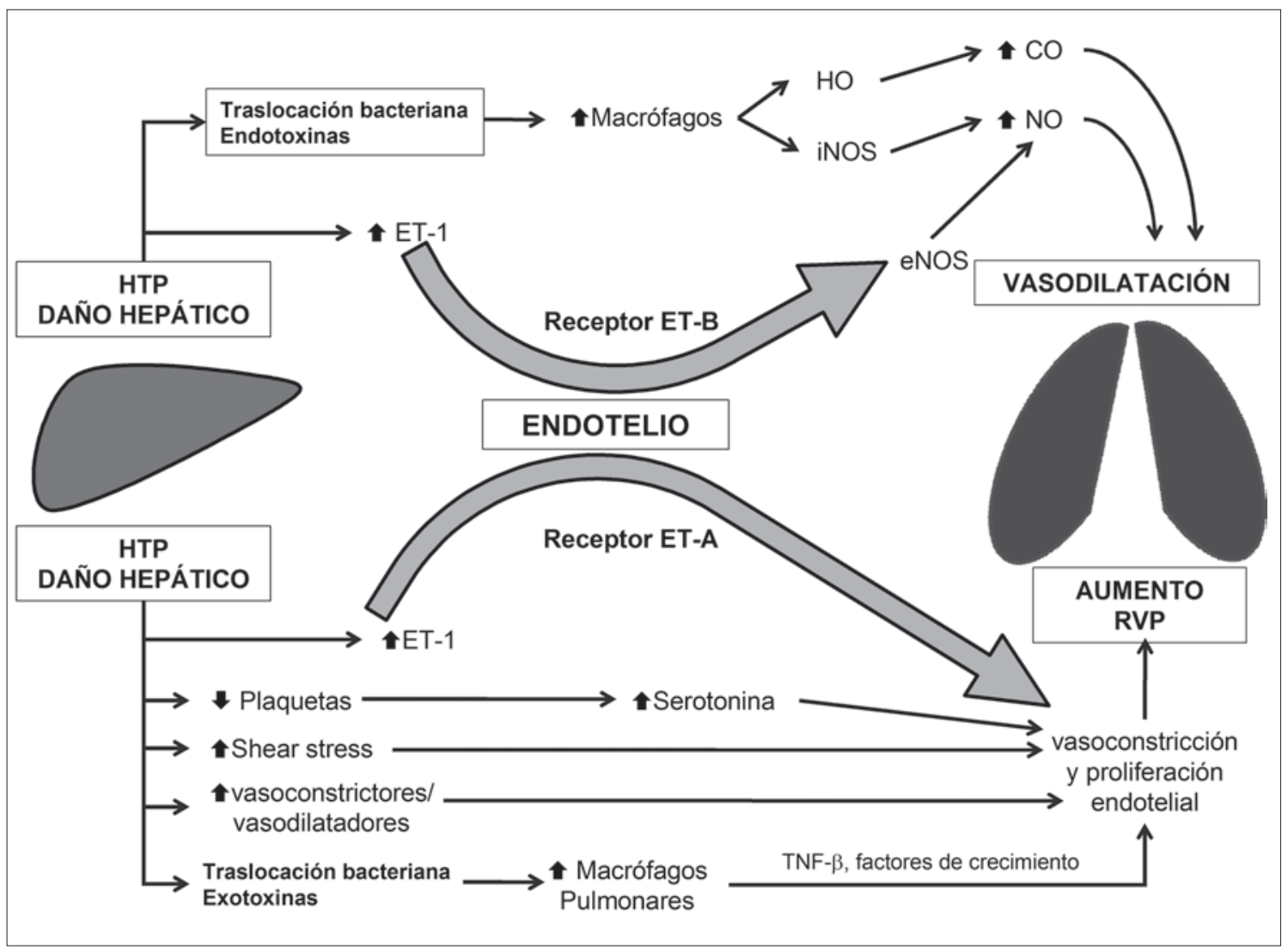

Figura 1. Esquema comparativo de hipótesis etiopatogénicas SHP e HPP. En la parte superior de la figura se ilustra la vía patogénica hipotética a partir de la asociación hipertensión portal y daño hepático crónico, por la cual se establece un estado de vasodilatación pulmonar en el SHP. En la parte inferior de la figura se ilustra la vía patogénica postulada para la HPP, en la que diferentes mediadores llevan a un aumento de la resistencia vascular pulmonar (RVP) (detalles en el texto). En el centro se resalta el papel del endotelio, el cual dependiendo del receptor de endotelina predominante gatillará una respuesta vasodilatadora o vasoconstrictora pulmonar. ET-1: endotelina-1; NO: óxido nítrico; CO: monóxido de carbono; HO: hemoxigenasa; iNOS: óxido nítrico sintetasa inducible; eNOS: óxido nítrico sintetasa endotelial; TNF$\beta$ : factor de necrosis tumoral beta. 


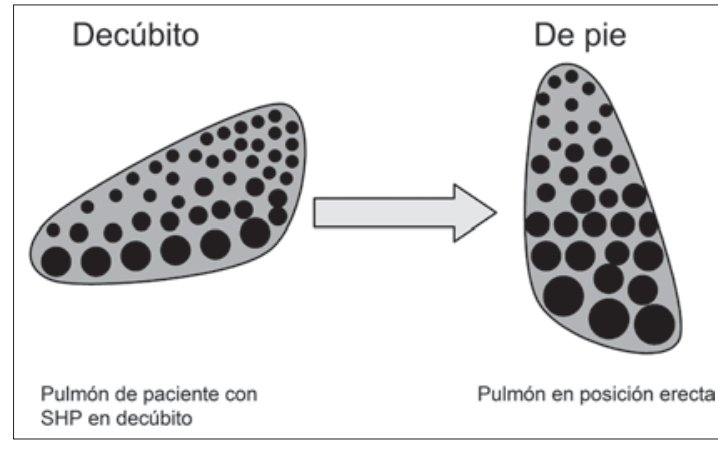

Figura 2. Representación esquemática del fenómeno de ortodeoxia y platipnea: al adoptar la posición erecta la mayor parte del flujo se dirige a las bases con un aumento de perfusión, sin un incremento proporcional de la ventilación.

modesto $(<10 \%)$ y raramente existe alteración de la difusión. En cambio, en estados más avanzados, todos los determinantes de la desoxigenación mencionados suelen coexistir. En definitiva, la difusión se encuentra severamente afectada por la intensa vasodilatación en el territorio pulmonar, asociada a la circulación hiperdinámica propia del estado cirrótico, y las modificaciones de ventilación-perfusión se expresan con mayor intensidad en relación a cambios posturales o a alteraciones parenquimatosas como atelectasias asociadas a la ascitis ${ }^{1,2,13}$.

\section{Clínica}

El síndrome se manifiesta típicamente por la presencia de disnea progresiva, que empeora en posición erecta y mejora con el decúbito (platipnea), en el contexto de un paciente con hipertensión portal o DHC. A lo anterior puede agregarse ortodeoxia en un $20-80 \%$ de los casos, cuya magnitud es proporcional a la gravedad del SHP, considerando que ésta también puede verse en otras patologías tales como embolia pulmonar recurrente, comunicación interauricular y post-neumonectomía ${ }^{14}$. En el examen físico, junto con los hallazgos propios del DHC, destacan la presencia de hipocratismo digital y telangiectasias aracniformes, que se describen como los marcadores físicos más sensibles ${ }^{1}$. En las etapas avanzadas, es frecuente encontrar cianosis de labios y lechos ungueales.

Cabe destacar que ante la sospecha clínica de SHP se debe descartar otras causas de disnea o hipoxemia mediante estudio de función pulmonar y de imágenes, ya que comorbilidades pulmonares crónicas tales como EPOC, asma o fibrosis pulmonar coexisten en alrededor de un tercio de los pacientes con $\mathrm{SHP}^{15}$.
Tabla 1. Nivel de gravedad en Síndrome hepatopulmonar e hipertensión portopulmonar

\begin{tabular}{lcc}
\hline & $\begin{array}{c}\text { Síndrome } \\
\text { hepato-pulmonar } \\
\mathbf{P a O}_{\mathbf{2}} \text { (mmHg) }\end{array}$ & $\begin{array}{c}\text { Hipertensión } \\
\text { portopulmunar } \\
\text { PAPM (mmHg) }\end{array}$ \\
\hline Leve & $>80$ & $25-35$ \\
Moderada & $60-80$ & $35-45$ \\
Grave & $50-60$ & $45-55$ \\
Muy grave & $<50$ & - \\
\hline
\end{tabular}

PAPM: presión arterial pulmonar media.

\section{Laboratorio}

Los pacientes con SHP presentan gases arteriales con hipoxemia comúnmente asociada a hipocapnia y aumento de $\mathrm{P}_{\mathrm{A}-\mathrm{a}} \mathrm{O}_{2}$ siendo esta última un marcador precoz de enfermedad ${ }^{2,6}$. La hipoxemia, elemento característico de este síndrome, permite clasificar a los pacientes según el nivel de gravedad (Tabla 1).

El estudio funcional espirométrico y de volúmenes pulmonares suele ser normal o levemente alterado y el estudio de difusión con monóxido de carbono (DLCO) puede estar alterado en grado moderado o severo, según el estadio del SHP. Mediante gasometría arterial u oximetría digital es posible documentar el fenómeno de ortodeoxia que caracteriza a esta condición ${ }^{1}$.

La radiografía de tórax suele ser normal ${ }^{1} . \mathrm{La}$ tomografía computada de tórax permite descartar condiciones pulmonares potencialmente responsables de la hipoxemia y puede evidenciar dilataciones en la circulación pulmonar periférica $^{2}$. El estudio angiográfico pulmonar puede mostrar un patrón de daño vascular focal o difuso, pero no se efectúa de rutina debido a la morbilidad asociada, baja disponibilidad y elevado costo $^{14}$.

\section{Diagnóstico}

El diagnóstico se plantea ante la presencia de DHC con hipertensión portal e hipoxemia, asociadas a shunt intrapulmonar el cual puede confirmarse mediante ecocardiografía de superficie con administración endovenosa de suero fisiológico agitado. En el paciente normal, las burbujas se verán sólo en las cavidades cardíacas derechas, ya que serán filtradas por la red capilar pulmonar. En pacientes con SHP, se observan burbujas en la aurícula izquierda luego de 3 a 6 latidos ${ }^{1}$, lo que sugiere la presencia de un shunt intrapulmonar y descarta uno intracardíaco en el cual las burbujas se visualizarían antes del tercer latido (Figuras 3, 4 y 5). 


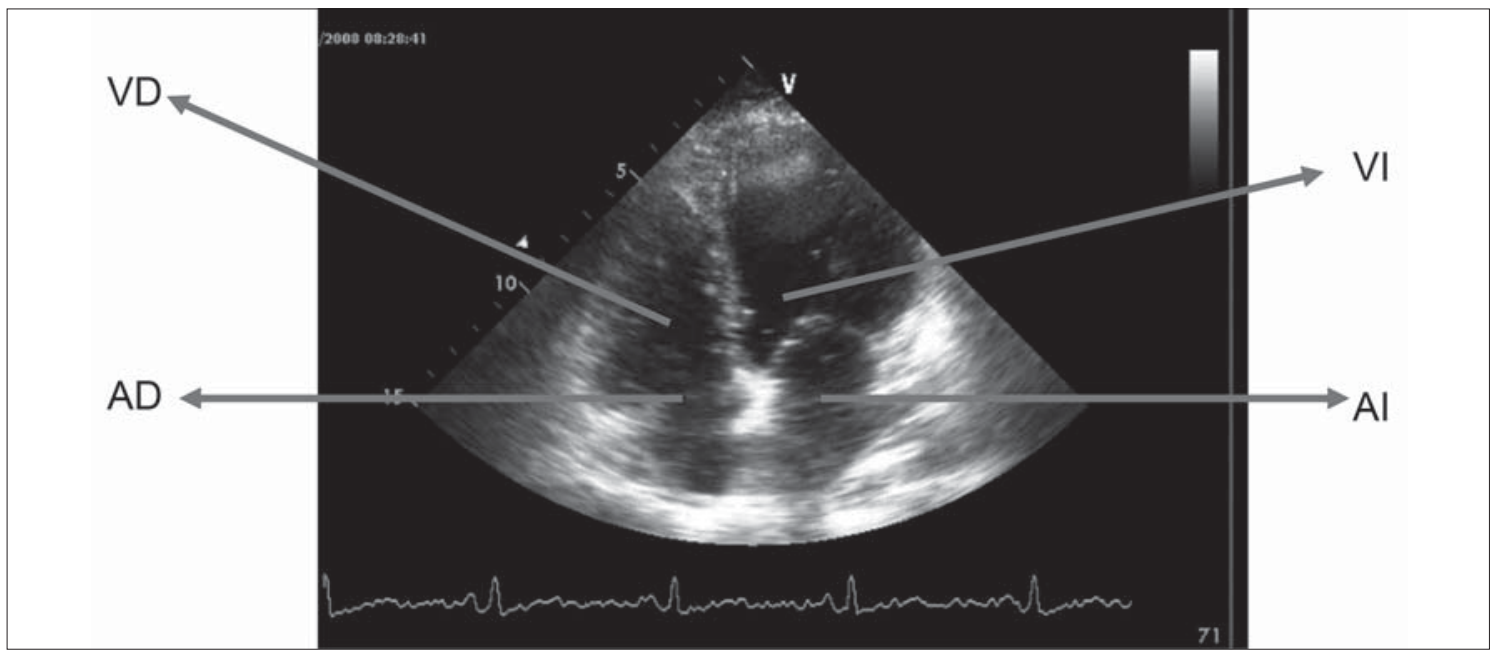

Figura 3. Imagen ecocardiográfica basal, transtorácica, que muestra una visión de las 4 cámaras antes de la inyección de suero salino agitado. AD: aurícula derecha; VD: ventrículo derecho; AI: aurícula izquierda; VD: ventrículo izquierdo.

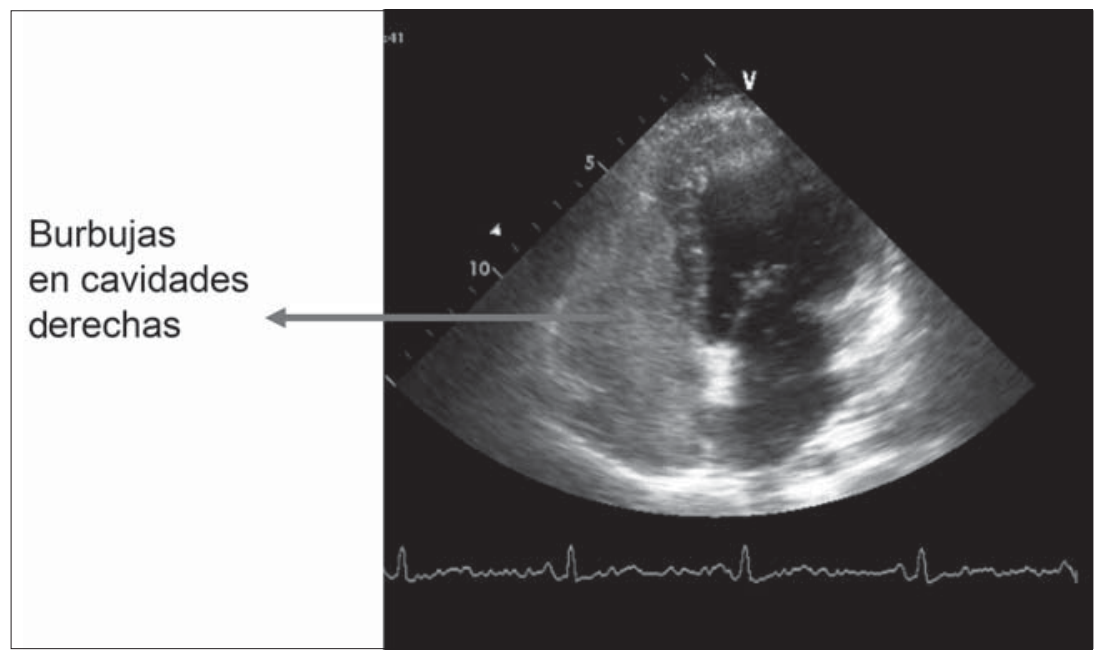

Figura 4. Visión ecográfica que demuestra la llegada de burbujas a las cavidades derechas ("contraste" en aurícula y ventrículo) luego del primer latido, es decir, precozmente a la administración de suero salino agitado.

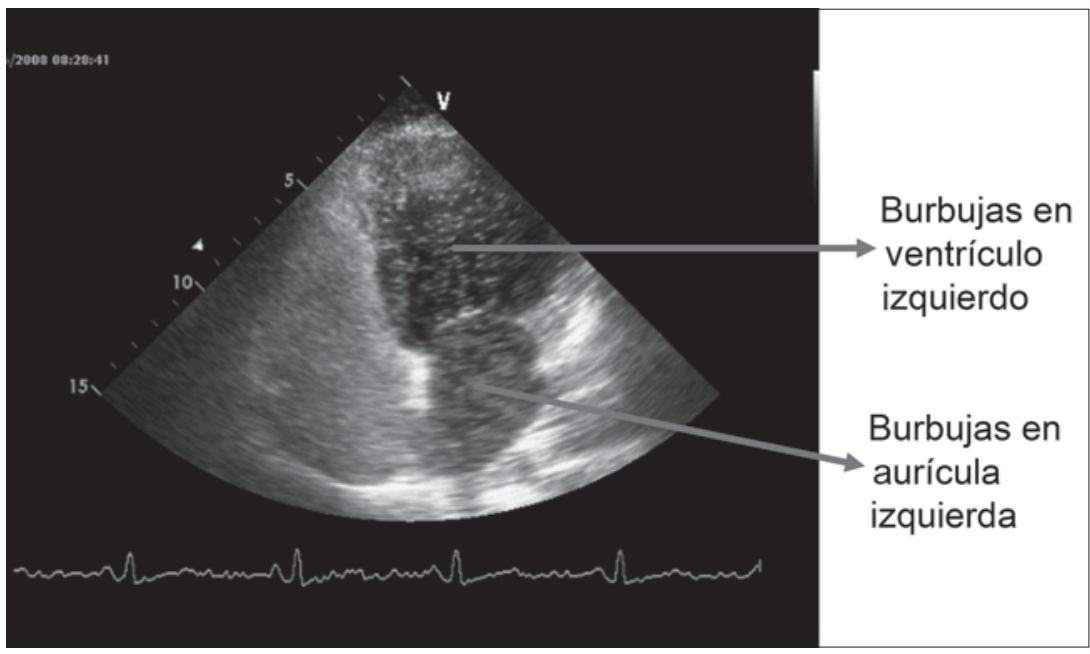

Figura 5. Visión ecográfica que demuestra la llegada de burbujas a las cavidades izquierdas ("contraste" en aurícula y ventrículo) luego del cuarto latido, es decir, tardíamente, propio de la presencia de un shunt extracardíaco, intrapulmonar. 
Si bien, la ecocardiografía transesofágica detecta shunts con mayor sensibilidad, no se justifica su uso rutinario por su costo, menor disponibilidad y mayores riesgos, por posibles várices esofágicas ${ }^{14}$. El estudio ecográfico además ofrece la posibilidad de detectar anomalías estructurales, evaluar función ventricular y descartar hipertensión pulmonar ${ }^{1}$.

Otra técnica para evidenciar la cuantía del shunt es la cintigrafía pulmonar usando macroagregados de albúmina marcada con $\mathrm{Tc}^{99 \mathrm{~m}}$. Normalmente los macroagregados de más de 20 $\mu \mathrm{m}$ son atrapados en la circulación pulmonar, pudiendo detectarse hasta un 5\% en tejidos extrapulmonares (shunt fisiológico). En el SHP, la detección periférica del marcador es mayor, pudiendo llegar hasta un $20 \%$. Este examen, sin embargo, no permite diferenciar entre un shunt intra o extrapulmonar ${ }^{1}$.

\section{Pronóstico y sobrevida}

El pronóstico es ominoso, ya que la sobrevida es de 16 a $38 \%$ a un año del diagnóstico y es aún más limitada si la $\mathrm{PaO}_{2}$ es $<50 \mathrm{mmHg}^{5}$. $\mathrm{La}$ presencia del SHP determina una menor sobrevida. En una serie de 111 pacientes, la sobrevida promedio de los pacientes con SHP fue de 10,6 meses, en comparación con una sobrevida de 40,8 meses en los pacientes cirróticos sin SHP ${ }^{16}$. Al efectuar el análisis de sobrevida basado en la severidad del daño hepático subyancente, la mortalidad se mantuvo más elevada en los pacientes con SHP, quienes fallecen por complicaciones del DHC e hipertensión portal, en estrecha correlación con la severidad de la hipoxemia. Otra serie mostró una sobrevida a 5 años de $23 \%$ ( 24 meses en promedio) en los pacientes con SHP no trasplantados respecto a 63\% (87 meses) en los con cirrosis hepática sin $\mathrm{SHP}^{17}$.

\section{Terapia}

El único tratamiento efectivo demostrado actualmente es el trasplante hepático $(\mathrm{TH})$, pero sólo un $10-20 \%$ de los pacientes son candidatos adecuados $^{18}$.

No existe terapia farmacológica que haya demostrado un beneficio significativo ni en el intercambio gaseoso ni en la sobrevida; sólo existen algunas series de casos que comunican discretos beneficios hemodinámicos y en el intercambio gaseoso con algunas drogas, como el azul de metileno que en infusión endovenosa continua inhibe los efectos del NO mediados por la guanilato ciclasa ${ }^{11}$. También se ha comunicado, en forma anecdótica, el uso del ajo en polvo administrado por 6 meses con mejoría parcial del intercambio gaseoso en 6 de 15 ca$\operatorname{sos}^{19}$. Por otra parte, son promisorios los estudios que surgen de la evaluación de otras drogas tales como norfloxacino y bloqueadores de las endotelinas. Sin embargo, por el momento no existe evidencia categórica que sustente el uso de ninguna terapia médica ${ }^{20}$.

El uso de los dispositivos que generan un shunt porto-sistémico por vía transyugular (TIPS) no ha logrado un rendimiento satisfactorio y permanece en un nivel experimental ${ }^{21}$. Tampoco existe suficiente evidencia del beneficio del oxígeno domiciliario que, sin embargo, se recomienda en aquellos con $\mathrm{PO}_{2}<60 \mathrm{mmHg}$ para evitar los efectos adversos circulatorios de la hipoxemia y mejorar subjetivamente la calidad de vida ${ }^{7}$. Como se ha señalado anteriormente, la única terapia efectiva que mejora el trastorno funcional circulatorio y la sobrevida es el trasplante hepático ${ }^{7}$, logrando un beneficio en el intercambio gaseoso en más del $85 \%$ de los trasplantados, modificaciones que pueden evidenciarse muchos meses luego del procedimiento ${ }^{17,18,22}$. En una serie reciente de la clínica Mayo se comunica una sobrevida post trasplante hepático a 1,3 y 5 años de 91,7-84,9 y 69,0\%, respectivamente ${ }^{17}$. Debe desatacarse que es fundamental la elección adecuada de los candidatos para $\mathrm{TH}$, ya que en pacientes con hipoxemia severa se ha observado una mortalidad postoperatoria tan elevada como $29 \%$, que puede disminuir a un $8,5 \%$ cuando la selección es más estricta $^{17,18}$. En relación a lo anterior, se debe preconizar la evaluación clínica y gasométrica seriada de los pacientes en espera de $\mathrm{TH}$ con el fin de realizar el procedimiento oportunamente y evitar factores post $\mathrm{TH}$ de mal pronóstico, tales como la hipoxemia severa $\left(\mathrm{PO}_{2}<50 \mathrm{mmHg}\right)$, el shunt $>20 \%$ y la edad avanzada. $\mathrm{La}^{\mathrm{PO}_{2}}$ es la principal herramienta en la toma de decisiones respecto al momento más adecuado para el $\mathrm{TH}$. Es así que se consideran a aquellos pacientes con $\mathrm{PO}_{2}$ entre 50-60 mmHg como los mejores candidatos para el TH y, en general, se excluye de dicho procedimiento a aquellos con $\mathrm{PO}_{2}$ inferior a $50 \mathrm{mmHg}$. En aquellos pacientes menos afectados funcionalmente, con $\mathrm{PO}_{2}$ entre 60-80 $\mathrm{mmHg}$ se sugiere una estricta vigilancia gasométrica y seguimiento periódico ${ }^{1,22}$. No obstante lo anterior, en el grupo con $\mathrm{PO}_{2}$ inferior a $50 \mathrm{mmHg}$, existen algunos casos con resultados exitosos post TH en que se observa una sobrevida superior a aquellos no trasplantados de la misma categoría gasométrica ${ }^{17}$. En relación a lo señalado previamente, es posible concluir que la decisión del TH en pacientes con SHP es compleja 
y exige un análisis caso a caso. No existe suficiente evidencia respecto al momento más adecuado para el procedimiento, ya que las series publicadas son pequeñas y el análisis de subgrupos no está exento de sesgos. Finalmente es importante destacar que la principal causa de mortalidad posterior a un $\mathrm{TH}$ no es de origen respiratorio sino que está fundamentalmente asociada con la falla o rechazo del injerto, sepsis, entre otras ${ }^{17,23}$.

\section{Hipertensión portopulmonar}

Esta entidad fue descrita por primera vez en 1951 por Mantz y Craige ${ }^{24}$, y se caracteriza por la presencia de hipertensión pulmonar, definida por aumento de la resistencia vascular pulmonar (RVP) en el territorio arteriolar, asociada a hipertensión portal, generalmente en el contexto de un paciente con enfermedad hepática subyacente ${ }^{1}$.

La hipertensión portopulmonar (HPP) afecta entre un $2-5 \%$ de los pacientes con cirrosis hepática ${ }^{25}$. La edad promedio de presentación es la quinta década de vida sin preferencia por género, mientras que la hipertensión pulmonar arterial (HPA) idiopática o asociada a mesenquimopatía se presenta con mayor frecuencia en mujeres en la cuarta década de vida ${ }^{26,27}$.

\section{Definición}

El diagnóstico de HPP se define por la asociación de HPA e hipertensión portal. La OMS en el consenso de expertos del $2003^{28}$ establece criterios hemodinámicos que definen esta condición a través del cateterismo derecho utilizando un catéter de Swan Ganz. El hallazgo de presión de arteria pulmonar media (PAPM) $>25$ $\mathrm{mmHg}$ en reposo, asociado a una presión del capilar pulmonar o presión de oclusión (PAOP) $<15 \mathrm{mmHg}$ y un aumento de la RVP > 240 dinas ${ }^{\bullet} \mathrm{s}^{\bullet} \mathrm{cm}^{-5}$ en concomitancia con hipertensión portal, permiten establecer el diagnóstico. Según este consenso, la HPP es una de las entidades que puede estar asociada a HPA, junto a enfermedades del tejido conectivo, shunts intracardíacos y VIH, entre otros, que deben diferenciarse de la HPA idiopática ${ }^{28}$.

\section{Etiopatogenia}

Los hallazgos histopatológicos a nivel microvascular pulmonar son indistinguibles de las otras formas de HPA y se caracterizan por los cambios obliterativos y proliferativos que conducen a un aumento de la resistencia en el territorio vascular pulmonar. A nivel arteriolar es posible apreciar la presencia de arteriopatía plexiforme, hipertrofia de la media, fibrosis de la intima, proliferación de la adventicia y necrosis fibrinoide. Asociado a lo anterior, se ha documentado la presencia de trombos y recanalización que resultan de trombosis in situ causada por injuria endotelial, agregación plaquetaria y un cierto estado de hipercoagulabilidad concomitante ${ }^{29}$.

Si bien la etiopatogenia de esta entidad no es completamente comprendida, existe certeza que el desarrollo de la HPP es independiente de cual sea la causa de la hipertensión portal ${ }^{4}$ y de la severidad de ésta, es decir, no existe correlación entre la severidad de la enfermedad hepática y la de la HPA ${ }^{1,2,4,30}$. Se postula que el daño microvascular arteriolar se debe a un desbalance entre los mediadores vasodilatadores y vasoconstrictores a favor de estos últimos, que causan daño endotelial y remodelación, proliferación del endotelio y del músculo liso y trombosis in situ. En relación a lo anterior, al aumentar el flujo sanguíneo pulmonar se produciría estrés en la pared vascular que activaría la cascada de eventos que lleva a los cambios histopatológicos de la $\mathrm{HPP}^{30}$. Sin embargo, éste no es el único factor causal y es probable que exista una interacción entre mediadores angiogénicos a nivel del endotelio y músculo liso. Se han comunicado en estos pacientes niveles elevados de ET-1, un potente vasoconstrictor de territorio pulmonar y hepático ${ }^{31}$, al cual se adjudica un papel patogénico, asociado a otros posibles desbalances de mediadores ${ }^{4}$. La presencia de shunt portosistémico permite el paso de componentes vasoactivos desde la circulación esplácnica a la circulación pulmonar eludiendo el metabolismo hepático ${ }^{25,32}$. Entre éstos se ha encontrado concentraciones elevadas de prostaglandinas F2 alfa, tromboxanos B2 y angiotensina $1^{30}$. También se ha documentado la presencia de niveles disminuidos de la sintetasa de prostaglandinas que son responsables de la vasodilatación en el territorio vascular pulmonar de estos pacientes, potenciando finalmente una interacción conjunta que lleva a una mayor vasoconstricción y proliferación del endotelio ${ }^{33}$.

\section{Clínica}

En estadios precoces la condición es asintomática y el síntoma inicial más frecuente es la disnea de esfuerzos que, por su carácter inespecífico obliga a efectuar el diagnóstico diferencial con otras condiciones asociadas al daño hepático crónico tales como miocardiopatía alcohólica, hidrotórax hepático y ascitis a tensión 
con atelectasias secundarias, mal acondicionamiento físico, emaciación muscular, etc ${ }^{5,25,34}$. En relación a la progresión de la enfermedad, pueden agregarse edema periférico, fatiga, hemoptisis, ortopnea, dolor torácico o síncope, siendo estos últimos de muy mal pronóstico vital ${ }^{2,5}$.

En el examen físico en condiciones más avanzadas, junto con los signos de DHC puede detectarse ingurgitación yugular, signos de disfunción de ventrículo derecho, acentuación del componente pulmonar del segundo ruido y un soplo holosistólico paraesternal izquierdo que traduce reflujo tricuspídeo ${ }^{2,5,25,34}$.

\section{Laboratorio}

La gasometría arterial suele mostrar una hipoxemia leve a moderada, un aumento $\mathrm{P}_{\mathrm{A}-\mathrm{a}} \mathrm{O}_{2}$ y una ligera hipocapnia de alrededor de 30 $\mathrm{mmHg}$. Los exámenes funcionales respiratorios pueden revelar una DLCO disminuida y una espirometría normal o restrictiva leve ${ }^{34}$.

En pacientes en etapa de evaluación para $\mathrm{TH}$, el hallazgo de HPA en la ecocardiografía bidimensional transtorácica tiene una sensibilidad de $97 \%$ y una especificidad de $77 \%$ para el diagnóstico de HPA moderada a severa ${ }^{35}$. En otras series, cuando se usa presión arterial sistólica pulmonar (PAPS) estimada mediante ecocardiografía con nivel de corte de $49 \mathrm{mmHg}$, la sensibilidad fue de $80 \%$, la especificidad de $96 \%$, el valor predictivo positivo de $60 \%$ y el negativos de $98 \%{ }^{36}$. Si bien la ecocardiografía es un excelente método de pesquisa, no logra distinguir entre HPP y aquella HP causada por estados hiperdinámicos, siendo el cateterismo derecho el examen de certeza ${ }^{30,35,37,38}$. Datos recientes sugieren que todo paciente enlistado para $\mathrm{TH}$ debe ser evaluado previamente con estudio ecocardiográfico y si éste muestra una PAPS $>50 \mathrm{mmHg}$, debe efectuarse la evaluación hemodinámica invasiva $^{38,39}$.

\section{Diagnóstico}

Generalmente, el diagnóstico de HPP es efectuado en promedio 4-7 años después del diagnóstico de hipertensión portal ${ }^{40}$, con o sin DHC, al aparecer disnea de esfuerzo y HPA en un estudio ecocardiográfico. El diagnóstico de certeza es hemodinámico invasivo mediante un cateterismo derecho y exige una PAPM $>25$ $\mathrm{mmHg}$ en reposo asociada a un aumento de la RVP en concomitancia con una presión de enclavamiento (PAOP) inferior a $15 \mathrm{mmHg}$. A los criterios anteriores se ha asociado, a manera de ayuda diagnóstica aunque sin acuerdo total de expertos, el hallazgo de un gradiente trans- pulmonar alterado $(>10 \mathrm{mmHg}$ ), que es la diferencia entre la PAPM y la PAOP. Este criterio no es esencial para el diagnóstico ni es consensual, pero puede ayudar en casos límites ${ }^{41}$. En relación al nivel de severidad de la HPP, que se ilustra en la Tabla 2, cabe destacar que éste no se correlaciona directamente con el nivel de daño hepático subyacente de modo que es posible el hallazgo de HPP en pacientes con diferentes grados de DHC, la cual tiene valor pronóstico en sí y en relación a la mortalidad asociada al $\mathrm{TH}^{25,42}$.

Es importante diferenciar la HPP de algunas enfermedades sistémicas que pueden afectar la circulación portal y pulmonar tales como el síndrome antifosfolípidos, la enfermedad mixta del tejido conectivo, la esquistosomiasis, la sarcoidosis, el lupus eritematoso sistémico, la anemia hemolítica microangiopática y el síndrome de inmunodeficiencia adquirida ${ }^{6}$. Sin embargo, ninguna de esas condiciones cumple los criterios hemodinámicos mencionados previamente que definen a la HPP. Por otro lado, si bien esta entidad normalmente está asociada a cirrosis hepática $^{1,2,4}$ en un grupo minoritario podría estar asociada a otras condiciones tales como: atresia de vía biliar, obstrucción extrahepática de vena porta, fibrosis portal no cirrótica, hipertensión portal sin cirrosis del lupus o fibrosis portal idiopática, todas ellas sin cirrosis ${ }^{25}$, enfatizando el hecho que lo que subyace a la HPP es fundamentalmente la hipertensión portal. En los pacientes con cirrosis la circulación es hiperdinámica, con elevado gasto cardíaco y reducida resistencia vascular sistémica ${ }^{2}$, lo que puede elevar PAPM manteniendo la RVP normal o baja, en contraste con la HPP en que la PAPM y RVP están elevadas ${ }^{4,40}$.

\section{Terapia}

No existe aún suficiente evidencia que sustente el tratamiento médico en esta entidad, debido a la falta de ensayos randomizados que evalúen las diferentes alternativas farmacológicas.

Se sugiere iniciar terapia cuando los pacientes son sintomáticos o presentan una PAPM > $35 \mathrm{mmHg}^{43}$ con RVP elevada. El tratamiento inicial se basa en diuréticos del tipo furosemida y/o espironolactona para disminuir la precarga ${ }^{1}$. Los TIPS no tienen rol en esta entidad y la anticoagulación no es recomendada en estos pacientes, a diferencia de otros grupos de HPA ${ }^{29,43}$, debido a que el trastorno de coagulación asociado al DHC significa un riego de hemorragia digestiva. Los bloqueadores de los canales de calcio están contraindicados ya que pueden au- 
Tabla 2. Tabla comparativa entre síndrome hepatopulmonar e hipertensión portopulmonar

\begin{tabular}{|c|c|c|}
\hline Característica diferencial & Síndrome hepatopulmonar & Hipertensión portopulmonar \\
\hline $\begin{array}{l}\text { Frecuencia en pacientes con daño } \\
\text { hepático crónico }\end{array}$ & $4-30 \%$ & $2-5 \%$ \\
\hline $\begin{array}{l}\text { Trastorno funcional y consecuencias } \\
\text { fisiopatológicas }\end{array}$ & $\begin{array}{l}\text { Shunt intrapulmonar } \\
\text { - Platipnea } \\
\text { - Ortodeoxia }\end{array}$ & $\begin{array}{l}\text { Aumento de la resistencia vascular } \\
\text { pulmonar } \\
\text { - Hipoxemia }\end{array}$ \\
\hline Gasometría arterial & Hipoxemia moderada a severa & Normal o con leve hipoxemia \\
\hline Síntomas y signos & $\begin{array}{l}\text { Disnea progresiva, cianosis, } \\
\text { hipocratismo digital, telangectasias } \\
\text { aracniformes }\end{array}$ & $\begin{array}{l}\text { Disnea progresiva, dolor torácico, } \\
\text { síncopes, sin cianosis, actividad del } \\
\text { VD, P2 aumentado, soplo tricuspídeo }\end{array}$ \\
\hline ECG & Inespecífico & $\begin{array}{l}\text { Bloqueo rama derecha, desviación eje } \\
\text { a derecha, hipertrofia VD }\end{array}$ \\
\hline Ecocardiograma de superficie & $\begin{array}{l}\text { Test de burbujas }(+) \\
\text { (contraste en aurícula izquierda } \\
\text { luego del } 3-6^{\circ} \text { latido) }\end{array}$ & $\begin{array}{l}\text { Test burbujas (-) } \\
\text { Hipertensión pulmonar } \\
\text { Crecimiento VD }\end{array}$ \\
\hline Hemodinamia & Normal con baja RVP & Elevada RVP, capilar normal \\
\hline Estudio de imágenes & Rx tórax normal & Cardiomegalia, ensanchamiento hiliar \\
\hline $\begin{array}{l}\text { Cintigrama con } \mathrm{Tc}^{99 \mathrm{~m}} \text { con } \\
\text { macro-agregados de albúmina }\end{array}$ & Shunt $>6 \%$ & Shunt $<6 \%$ \\
\hline Angiografía pulmonar & Normal o con CAV & $\begin{array}{l}\text { Grandes arterias pulmonares, } \\
\text { "prunning" arterial distal }\end{array}$ \\
\hline Terapia & Trasplante hepático & $\begin{array}{l}\text { Sildenafil, prostaciclinas, antiendote- } \\
\text { linas. Trasplante hepático sólo está } \\
\text { indicado con PAPM }<35 \mathrm{mmHg}\end{array}$ \\
\hline
\end{tabular}

VD: ventrículo derecho. P2: componente pulmonar segundo tono cardíaco. Tc ${ }^{99 \mathrm{~m}}$ : Tecnecio 99 metaestable (radiofármaco usado para la cintigrafía pulmonar en medicina nuclear). RVP: resistencia vascular pulmonar. CAV: Comunicaciones arterio-venosas. Prunning: poda o adelgazamiento vascular. PAPM: Presión arteria pulmonar media. Modificada de referencia 1.

mentar el gradiente venoso hepático ${ }^{44}$, exacerbar la vasodilatación mesentérica y aumentar el flujo a la circulación portal, lo que pudiera facilitar el desarrollo de várices gastro-esofágicas. Tampoco se recomienda el uso de beta bloqueadores ya que pudieran deteriorar la capacidad de ejercicio y la hemodinamia pulmonar ${ }^{45}$. El epoprostenol endovenoso, una prostaciclina sintética, es la droga mejor estudiada en HPP y ha demostrado disminuir la PAPM en el corto y largo plazo ${ }^{30,46}$. No queda claro su beneficio en la sobrevida, pero existen algunos estudios que demuestran que en pacientes seleccionados, en concomitancia con el $\mathrm{TH}$, mejoraría la sobrevida $^{41}$. Esta es una droga costosa, no exenta de efectos adversos y que requiere para su uso una bomba de infusión continua lo que hace complicada su utilización masiva.

El bosentan, antagonista dual de los receptores ET-A y B, aprobado por la FDA en 2002 para el tratamiento de la HPA, pudiera ser una alternativa interesante y existen estudios prometedores, aunque tiene documentada toxicidad hepática $^{47}$.

También se ha utilizado con algún éxito el sildenafil, un inhibidor selectivo de la fosfodiesterasa 5, que se encuentra ampliamente distribuida a nivel pulmonar. Esta inhibición facilita la acción vasodilatadora del NO a nivel arteriolar pulmonar, disminuye la presión y resistencia vascular con la consiguiente mejoría del débito cardíaco. Esta es una droga aprobada por la FDA para HPA, segura y accesible con resultados beneficiosos en pequeñas series clínicas para pacientes con $\mathrm{HPP}^{48}$. La terapia combinada parece ser una mejor alternativa, pero se encuentra en espera de evidencia. El TH fue considerado por mucho tiempo una contraindicación en presencia de HPP, sin embargo hoy, en pacientes altamente seleccionados, es una alternativa factible y efectiva. Debido a que la presencia de $\mathrm{HPP}$ en un paciente seleccionado para $\mathrm{TH}$ 
incrementa la morbimortalidad en el período perioperatorio y a largo plazo $^{1,49}$, los criterios para incorporar pacientes a las listas de $\mathrm{TH}$ se han restringido a aquellos con una HPA leve. Una PAPM > $50 \mathrm{mmHg}$ medida durante el cateterismo derecho, se considera una contraindicación absoluta para $\mathrm{TH}^{1}$. Por otro lado, para aquellos candidatos a TH con PAPM $>35 \mathrm{mmHg}$, que acceden a terapia médica específica, el objetivo de ésta es reducir la PAPM a $<35 \mathrm{mmHg}$ y la resistencia vascular pulmonar a $<400$ dinas $\mathrm{S} \mathrm{cm}^{-5}$ antes del $\mathrm{TH}^{41,42,49}$. Esta aproximación se encuentra sustentada por la mejor evolución que presentan aquellos pacientes que cursan con una $\mathrm{PAPM}<35 \mathrm{mmHg}$ en el preoperatorio de $\mathrm{TH}^{42}$. En la serie de la clínica Mayo, la mortalidad posterior al trasplante hepático fue de 100\% cuando la PAPM excedía los $50 \mathrm{mmHg}$ y de $50 \%$ cuando se encontraba entre 35 y $50 \mathrm{mmHg}^{42}$. Es importante destacar que posterior a un $\mathrm{TH}$ exitoso, si bien existe mejoría hemodinámica, no se ha visto regresión del daño endotelial ${ }^{5}$ sino sólo estabilización funcional. La combinación de trasplante hepático y pulmonar no ha sido evaluada y podría ser objeto de estudios futuros. Se ha comunicado que el epoprostenol juega un rol relevante como un puente al TH logrando una estabilización hemodinámica suficiente como para permitir que hasta un $75 \%$ de los pacientes puedan ser enlistados para $\mathrm{TH}^{50}$. La resolución de los síntomas post TH puede ser lenta, de meses o años ${ }^{25,42}$. Si bien la evolución post $\mathrm{TH}$ suele ser favorable, se ha reportado en algunos casos tanto persistencia, progresión o recurrencia de la $\mathrm{HPP}^{51}$.

\section{Pronóstico}

En comparación con la HPA idiopática, los pacientes con HPP tienen una mejor sobrevida $^{4,29,43}$ lo que se ilustra en un estudio retrospectivo que, cabe destacar, es previo al advenimiento de las prostaciclinas, en que evidenció una sobrevida de 57 meses para los pacientes con HPP comparado con 31 en el grupo idiopático $^{30}$.

En la serie de la Clínica Mayo se reporta una sobrevida a 1, 2 y 5 años en pacientes con HPP de $71 \%, 58 \%$ y $44 \%$ respectivamente, independiente del estado de la enfermedad ${ }^{52}$.

\section{Conclusión}

Al momento de evaluar un paciente con daño hepático crónico, hipertensión portal y disnea, con o sin hipoxemia, se sugiere descartar las causas habituales de compromiso funcional respiratorio que pueden asociarse al daño hepático. Luego de ello efectuar una ecocardiografía con inyección de burbujas y medición de presión de arteria pulmonar.

Si se confirma el diagnóstico de síndrome hépato-pulmonar, la incorporación a un programa de trasplante deberá ser inmediata, ya que no existe otra terapia comprobada disponible.

Si el estudio ecocardiográfico demuestra HPA, de no mediar contraindicación, es adecuado efectuar un estudio hemodinámico confirmatorio. Si éste es concluyente, el paciente debería recibir alguna terapia específica ya sea a base de prostaciclinas, sólo disponibles en nuestro país para uso mediante nebulizador (Iloprost) o inhibidores de la fosfodiesterasa (sildenafil). El TH se reserva para los casos con HPP leve, con PAPM < $35 \mathrm{mmHg}$ ya sea basal o post tratamiento.

De acuerdo a lo expuesto, los principales elementos que pueden ayudar al clínico a identificar y diferenciar ambas entidades se resumen en la Tabla 2 y en el apéndice se muestran 3 casos clínicos ilustrativos.

\section{Agradecimientos}

Agradecemos al Dr. Claudio Parra, cardiólogo jefe del laboratorio de ecocardiografía del Hospital del Salvador, por su gentileza en aportarnos las imágenes ecográficas que ilustran el test de inyección de suero salino agitado (test de burbujas) para la evaluación de shunt.

\section{Bibliografía}

1.- RODRÍGUEZ-ROISIN R, KROWKA M J, HERVÉ P, FALLON M. Pulmonary-Hepatic Vascular Disorders (PHD). Eur Respir J 2004; 24: 861-80.

2.- HERVE P, LE PAVEC J, SZTRYMF B, DECANTE B, SAVALE L, SITBON O. Pulmonary vascular abnormalities in cirrhosis. Best Pract Res Clin Gastroenterol 2007; 21: 141-59.

3.- FLUCKIGER M. Vorkommen von trommelschagelformigen finger-end phalagen ohne chronische veranderungen an der lungen oder am herzen. Wein Med Wehnschr 1884; 49: 1457.

4.- HOEPER M, KROWKA M, STRASSBURG C. Portopulmonary hypertension and hepatopulmonary syndrome. Lancet 2004; 303: 1461-68.

5.- NAEIJE R. Hepatopulmonary syndrome and portopulmonary hypertension. Swiss Med Wkly 2003; 133: 163-9.

6.- SCHENK P, FUHRMANN V, MADL C, FUNK S, LEHR, KANDEL O, et al. Hepatopulmonary syndrome: prevalence and predictive value of various cut offs for arterial oxygenation and their clinical consequences. Gut 2002; 51: 853-9. 
7.- PALMA D, FALLON M. The Hepatopulmonary Syndrome. J Hepatol 2006; 45: 617-25.

8.- DINH-XUAN A T, NAEIJE R R. The hepatopulmonary syndrome: NO way out? Eur Respir J 2004; 23: 661-2.

9.- ROLLA G, BRUSSINO L, COLAGRANDE P, SCAPPATICCI E, MORELLO M, BERGERONE S, et al. Exhaled nitric oxide and impaired oxygenation in cirrhotic patients before and after liver transplantation. Ann Intern Med 1998; 129: 375-3.

10.- GÓMEZ F, BARBERA J, ROCA J, BURGOS F, GISTAU C, RODRÍGUEZ-ROISIN R. Effects of Nebulized NGnitro-L-arginine Methyl Ester in Patients With Hepatopulmonary Syndrome. Hepatology 2006; 43: 1084-91.

11.- SCHENK P, MADL C, REZAIE-MAJD S, LEHR S, MULLER C. Methylene Blue Improves the Hepatopulmonary Syndrome. Ann Intern Med 2000; 133: 701-6.

12.- ARGUEDAS M R, DRAKE B B, KAPOOR A, FALLON M B. Carboxyhemoglobin levels in cirrhotic patients with and without hepatopulmonary syndrome. Gastroenterology 2005; 128: 328-33.

13.- GÓMEZ F, MARTÍNEZ-PALLI G, BARBERA J, ROCA J, NAVASA M, RODRÍGUEZ-ROISIN R. Gas exchange mechanism of orthodeoxia in hepatopulmonary syndrome. Hepatology 2004; 40: 660-6.

14.- VARGHESE J, ILIAS-BASHA H, DHANASEKARAN R, SINGH S, VENKATARAMAN J. Hepatopulmonary syndrome-Past to present. Annals of Hepatology 2007; 6: $135-42$.

15.- MARTÍNEZ G, BARBERA J A, NAVASA M, ROCA J, VISA J, RODRÍGUEZ-ROISIN R. Hepatopulmonary syndrome associated with cardiorespiratory disease. J Hepatol 1999; 30: 882-9.

16.- SCHENK P, SCHONIGER-HEKELE M, FUHRMANN V, MADL C, SILBERHUMER G, MULLER C. Prognostic significance of the hepatopulmonary syndrome in patients with cirrhosis. Gastroenterology 2003; 125: 1042-52.

17.- SWANSON K, WIESNER R, KROWKA M. Natural history of hepatopulmonary syndrome: Impact of liver transplantation. Hepatology 2005; 41: 1122-9.

18.- MARTÍNEZ G P, BARBERA J A, VISA J, RIMOLA A, PARE J C, ROCA J, et al. Hepatopulmonary syndrome in candidates for liver transplantation. J Hepatol 2001; 34: 651-7.

19.- ABRAMS G A, FALLON M B. Treatment of hepatopulmonary syndrome with Allium sativum (garlic): a pilot trial. J Clin Gastroenterol 1998; 27: 232-5.

20.- ANEL R M, SHEAGREN J N. Novel presentation and approach to management of hepatopulmonary syndrome with use of antimicrobial agents. Clin Infect Dis 2001; 32: E131-6.

21.- MARTÍNEZ-PALLI G, DRAKE B, GARCÍA-PAGAN J, BARBERA J, ARGUEDAS M, RODRÍGUEZ-ROISIN $\mathrm{R}$, et al. Effect of transjugular intrahepatic portosystemic shunt on pulmonary gas exchange in patients with portal hypertension and hepatopulmonary syndrome. World J Gastroenterol 2005; 11: 6858-6.

22.- PASTOR C M, SHIFFER E. Therapy Insight: hepatopulmonary síndrome and orthotopic liver transplantation. Nat Clin Pract Gastroenterol Hepatol 2007; 4: 614-21.

23.- ARGUEDAS M, ABRAMS G, KROWKA, FALLON M. Prospective evaluation of outcomes and predictors of mortality in patients with hepatopulmonary syndrome undergoing liver transplantation. Hepatology 2003; 37: 192-7.

24.- MANTZ F A, CRAIGE E. Portal axis thrombosis with spontaneous porto-caval shunt and resultant cor pulmonale. Arch Pathol 1951; 52: 91-7.

25.- BUDHIRAJA R, HASSOUN P M. Portopulmonary hypertension: a tale of two circulations. Chest 2003; 123: 562-76.

26.- RICH S, DANTZKER D R, AYRES S M, BERGOFSKY E H, BRUNDAGE B H, DETRE K M, et al. Primary pulmonary hypertension. A national prospective study. Ann Intern Med 1987; 107: 216-23.

27.- KROWKA M J, CORTESE D A. Hepatopulmonary syndrome. Current concepts in diagnosis and therapeutic considerations. Chest 1994; 105: 1528-37.

28.- SIMONNEAU G, GALIE N, RUBIN LJ, LANGLEBEN D, SEEGER W, DOMENIGHETTI G, et al. Clinical classification of pulmonary hypertension. J Am Coll Cardiol 2004; 43: 5S-12S.

29.- RUBIN L J, BADESCH D B. Evaluation and management of the patient with pulmonary arterial hypertension. Ann Intern Med 2005; 143: 282-92.

30.- HERVE P, LEBREC D, BRENOT F, SIMONNEAU G, HUMBERT M, SITBON O, et al. Pulmonary vascular disorders in portal hypertension. Eur Respir J 1998; 11: 1153-66.

31.- MOORE K, WENDON J, FRAZER M, KARANI J, WILLIAMS R, BADR K. Plasma endothelin immunoreactivity in liver disease and the hepatorenal syndrome.N Engl J Med 1992; 327: 1774-8.

32.- MANDELL M S, GROVES B M. Pulmonary hypertension in chronic liver disease. Clin Chest Med 1996; 17: 17-33.

33.- TUDER R M, COOL C D, GERACI M W, WANG J, ABMAN S H, WRIGHT L, et al. Prostacyclin synthase expression is decreased in lungs from patients with severe pulmonary hypertension. Am J Respir Crit Care Med 1999; 159: 1925-32.

34.- GOLBIN J M, KROWKA M J. Portopulmonary hypertension; en Palevsky H, Clinic in Chest Medicine, Philadelphia, W.B. Saunders Company 2007; 20318.

35.- KIM W R, KROWKA M J, PLEVAK D J, LEE J, RETTKE S R, FRANTZ R P, et al. Accuracy of doppler echocardiography in the assessment of pulmonary hypertension in liver transplant candidates. Liver Transpl 2000; 6: 453-8.

36.- COLLE I O, MOREAU R, GODINHO E, BELGHITI J, ETTORI F, COHEN-SOLAL A, et al. Diagnosis of portopulmonary hypertension in candidates for liver transplantation: a prospective study. Hepatology 2003; 37: 401-9.

37.- KROWKA M J. Portopulmonary hypertension: diagnostic advances and caveats. Liver Transpl 2003; 9: 1336-7.

38.- COTTON C L, GANDHI S, VAITKUS P T, MASSAD M G, BENEDETTI E, MRTEK R G, et al. Role of echocardiography in detecting portopulmonary hypertension in liver transplant candidates. Liver Transpl 2002; 8: 1051-4.

39.- KROWKA M J, SWANSON K L, FRANTZ R P, McGOON M D, WIESNER R H. Portopulmonary hypertension: results from a 10-year screening algorithm. Hepatology 2006; 44: 1502-10.

40.- HADENGUE A, BENHAYOUN M K, LEBREC D, BENHAMOU J P. Pulmonary hypertension complicating portal hypertension: prevalence and relation to splanchnic hemodynamics. Gastroenterology 1991; 
100: $520-8$.

41.- KROWKA M J. Evolving dilemmas and management of portopulmonary hypertension. Semin Liver Dis 2006; 26: 265-72.

42.- KROWKA M J, PLEVAK D J, FINDLAY J Y, ROSEN C B, WIESNER R H, KROM R A. Pulmonary hemodynamics and perioperative cardiopulmonaryrelated mortality in patients with portopulmonary hypertension undergoing liver transplantation. Liver Transpl 2000; 6: 443-50.

43.- HOEPER M M, RUBIN L J. Update in pulmonary hypertension 2005. Am J Respir Crit Care Med 2006; 173: 499-505.

44.- OTA K, SHIJO H, KOKAWA H, KUBARA K, KIM T, AKIYOSHI N, et al. Effects of nifedipine on hepatic venous pressure gradient and portal vein blood flow in patients with cirrhosis. J Gastroenterol Hepatol 1995; 10: 198-204.

45.- PROVENCHER S, HERVE P, JAIS X, LEBREC D, HUMBERT M, SIMONNEAU G, et al. Deleterious effects of beta-blockers on exercise capacity and hemodynamics in patients with portopulmonary hypertension. Gastroenterology 2006; 130: 120-6.

46.- KUO P C, JOHNSON L B, PLOTKIN J S, HOWELL C D, BARTLETT S T, RUBIN L J. Continuous intravenous infusion of epoprostenol for the treatment of portopulmonary hypertension. Transplantation
1997; 63: 604-6.

47.- HOEPER M M, HALANK M, MARX C, HOEFFKEN G, SEYFARTH H J, SCHAUER J, et al. Bosentan therapy for portopulmonary hypertensin. Eur Respir J 2005; 25: 502-8.

48.- REICHENBERGER F, VOSWINCKEL R, STEVELING E, ENKE B, KRECKEL A, OLSCHEWSKI H, et al. Sildenafil treatment for portopulmonary hypertension. Eur Resp J 2006; 28: 563-7.

49.- MURRAY K F, CARITHERS R L Jr. AASLD practice guidelines: evaluation of the patient for liver transplantation. Hepatology 2005; 41: 1407-32.

50.- SUSSMAN N, KAZA V, BARSHES N, STRIBLING R, GOSS J, O'MAHONY C, et al. Successful liver transplantation following medical management of portopulmonary hypertension: a single-center series. Am J Transplant 2006; 6: 2177-82.

51.- KETT D H, ACOSTA R C, CAMPOS M A, RODRÍGUEZ M J, QUARTIN A A, SCHEIN R M. Recurrent portopulmonary hypertension after liver transplantation: management with epoprostenol and resolution after retransplantation. Liver Transpl 2001; 7: $645-8$

52.- GOLBIN J M, KROWKA M J. Portopulmonary hypertension. En: Palevsky HI eds. Clinic in Chest Medicine. Philadelphia US. WB Saunders Company 2007; 203-18.

\section{Apendice}

\section{Casos Clínicos}

Caso 1: Paciente A.V.C de 19 años, de sexo femenino, portadora de una cirrosis biliar secundaria a un quiste coledociano operado a la edad de 9 años. Evoluciona con disnea lentamente progresiva que motiva su consulta en el Instituto Nacional del Tórax a la edad de 18 años. En el examen físico destacaba una paciente sin disnea en reposo, pero con saturación en oximetría digital de $72 \%$ en posición sentada y de $84 \%$ en decúbito supino, asociada a cianosis de labios y lechos ungueales e hipocratismo digital. A la auscultación pulmonar no se registraron ruidos anormales ni soplos. Su radiografía de tórax era normal.

En su evaluación funcional, la espirometría mostraba una alteración restrictiva leve, con alteración difusional severa (DLCO corregido para hematocrito de $51 \%$ : $13,24 \mathrm{ml} / \mathrm{min} / \mathrm{mmHg}, 43 \%$ del predictivo). La gasometría arterial mostró una severa hipoxemia, con $\mathrm{PaO}_{2}$ de $49 \mathrm{mmHg}$, saturación arterial de 86,4\%, $\mathrm{PaCO}_{2}$ de $29 \mathrm{mmHg}$ y pH 7,4 con aire ambiental. Luego de 20 minutos de ventilación con oxígeno al $100 \%$ mediante mascarilla hermética, se observó una mejoría de la oxemia a una $\mathrm{PaO}_{2}$ de $122,4 \mathrm{mmHg}$, y saturación de 98,6\%. En relación a lo anterior se calculó un shunt de aproximadamente 23\%. El estudio mediante ecocardiografía de superficie con test de burbujas fue sugerente de shunt extracardíacointrapulmonar, sin hipertensión pulmonar, con fracción de eyección conservada.

Si bien se consideró una candidata para un tras- plante hepático se decidió, en atención a su edad y adecuada condición general y psíquica, efectuar una terapia de prueba, absolutamente empírica, a base de broncodilatadores de acción prolongada asociados a kinesiterapia respiratoria. En la evaluación al mes de esta terapia, no se observaron cambios significativos del punto de vista clínico y gasométrico.

En conclusión se trata de un SHP severo y su caso está en evaluación por el comité de trasplante hepático.

Comentario: Este caso es ilustrativo de una paciente con un grave compromiso funcional lo que restringe las alternativas terapéuticas y aleja la opción de trasplante por los riesgos perioperatorios asociados al nivel de hipoxemia de la paciente.

Caso 2: Paciente TPJ de 61 años, sexo femenino, con antecedentes de diabetes mellitus tipo 2 e hipertensión arterial crónica. Consulta en 2001 por disnea progresiva y tos de un año de evolución. Los exámenes funcionales demuestran gasometría arterial en reposo y ejercicio, espirometría y capacidad de difusión normales. La ecocardiografía fue normal. Su evolución se complica con hemorragia digestiva alta debida a várices esofágicas grado IIIII que son objeto de escleroterapia y ligadura. Los exámenes de laboratorio y ecografía abdominal demuestran daño hepático crónico de causa no precisada e hipertensión portal, Child-Pugh B. Recibe tratamiento con propranolol y espironolactona. El estudio serológico (consistente en ANA, Ac. antimitocondriales, anticuerpos antimúsculo liso, fac- 
tor reumatoideo y ENA) fue negativo. En 2005 vuelve a consultar para control en el policlínico respiratorio con cuadro de baja de peso y disnea. Entre los exámenes destaca una TAC de tórax con signos de hipertensión pulmonar, sin embolia ni aguda ni crónica, sin daño parenquimatoso pulmonar y con esplenomegalia. En 2006 se documenta mediante ecocardiograma una presión arterial sistólica pulmonar elevada de $58 \mathrm{mmHg}$ con test de burbujas negativo. Ante la fuerte sospecha de hipertensión portopulmonar (HPP) se programa cateterismo cardíaco para su confirmación, pero la paciente fallece a consecuencia de su falla hepática antes de efectuarse el examen.

Caso 3: Paciente M.A.L, de sexo femenino que consulta a la edad de 52 años por fatiga crónica. En la evaluación inicial se documenta colelitiasis y es programada para cirugía, en la que se toma biopsia hepática cuyo informe histológico concluye cirrosis biliar primaria en etapa II/IV, iniciándose tratamiento con ácido ursodeoxicólico. Ocho años después (2002) presenta encefalopatía recurrente, progresando su daño hepático a clase funcional Child-Pugh C y MELD 16. Fue enlistada para el programa de trasplante hepático el 10/11/2004 a la edad de 62 años. Durante 2005 se efectuó ecocardiografía que estimó una presión de arteria pulmonar normal y buena función cardíaca.

En septiembre de 2006 ingresa a pabellón para trasplante hepático y en el cateterismo derecho con catéter de Swan Ganz preoperatorio se evidencia una PAPS de $90 \mathrm{mmHg}$ y PAPM de $60 \mathrm{mmHg}$, con presión capilar pulmonar menor de $15 \mathrm{mmHg}$. A pesar de los riesgos asociados se decide realizar el trasplante hepático de común acuerdo con la paciente y su familia. En el postoperatorio en la UTI, a la terapia inmunosupresora con esteroides y ciclosporina se agrega Sildenafil $50 \mathrm{mg}$ cada 6 horas para estabilizar la HPP. El perfil hemodinámico del $5^{\circ}$ día post trasplante mostró una PAPM de 50 $\mathrm{mmHg}$. La ecocardiografía detectó una leve insuficiencia tricuspídea. La paciente inicia terapia anticoagulante como parte de la terapia de su HPP y de una trombosis de una rama de la vena porta in situ detectada durante la cirugía. Su evolución fue favorable, con capacidad funcional I de modo que es dada de alta a las tres semanas post trasplante.

La ecocardiografía de control a los tres meses post trasplante mostró una PAPS de $60 \mathrm{mmHg}$ y PAPM de $35 \mathrm{mmHg}$. Ante esta evolución favorable se mantiene terapia con sildenafil, anticoagulación y furosemida, asociados a la terapia inmunosupresora. El control ecocardiográfico del sexto mes fue similar al previo, con una buena función sistólica global pero fallece a los diez meses post trasplante debido a una sepsis secundaria a infección pulmonar por nocardia.

Comentario: Los dos últimos casos presentados son ilustrativos de la evolución que pueden seguir los pacientes con HPP ya sea que por su gravedad no alcancen a completar su estudio pretrasplante o, que pese a efectuarse el TH, sufran una complicación inherente a la terapia inmunosupresora.

Correspondencia a:

Dra. Mónica Zagolín B.

Instituto Nacional del Tórax.

Av. José Manuel Infante 717, Santiago, Chile.

E-mail: mzagolin@torax.cl 